\title{
Evaluation of cytokines and soluble adhesion molecules in patients with newly diagnosed acute myeloid leukemia: the role of TNF-alpha and FLT3-ITD
}

\author{
Tomas Kupsa ${ }^{\mathrm{a}, \mathrm{b}}$, Jan Vanekc, Martina Vasatovad ${ }^{\mathrm{d}}$ Iva Karesovad ${ }^{\mathrm{d}}$, Pavel Zak ${ }^{\mathrm{b}}$, Ladislav Jebavy ${ }^{\mathrm{a}, \mathrm{b}}$, Jan M. Horacek ${ }^{\mathrm{a}, \mathrm{b}}$
}

\begin{abstract}
Objectives. Acute myeloid leukemia (AML) cells are highly resistant to therapy. The presumed molecular basis of this resistance is the effect of tumor necrosis factor alpha (TNF- $a$ ) and other cytokines on endothelial adhesion molecule expression. The aim of this study was to test the hypothesis that cytokines and soluble adhesion molecules correlate in AML.

Methods. Baseline serum levels of 17 cytokines and 5 soluble adhesion molecules were measured in 53 AML patients using biochip array technology. Age, leukocyte count, secondary AML, CRP, FLT3-ITD and remission were variables. Statistical analysis was performed in R version 3.1.2.

Results. VCAM-1 correlated with ICAM-1 $(P<0.0001)$, E-selectin $(P<0.0001)$, leukocyte count $(P=0.0005)$ and TNF-a $(P$ $=0.0035)$. E-selectin correlated with leukocyte count $(P<0.0001)$, P-selectin $(P=0.0032)$ and MCP-1 $(P=0.0119)$. CRP correlated with IL-6 $(P<0.0001)$, leukocyte count negatively correlated with IL-7 $(P=0.0318)$. FLT3-ITD was associated with higher E-selectin $(P=0.0010)$ and lower IL-7 $(P=0.0252)$. Secondary AML patients were older. Failure of induction therapy was associated with significantly higher CRP and lower P-selectin. Leukocyte count $(P<0.0001)$, FLT3-ITD $(P=$ 0.0017) and secondary AML $(P=0.0439)$ influenced the principal component.

Conclusions. Leukemic cells can modulate the microenvironment. Cytokine, adhesion molecule levels and leukocyte count correlate in AML. Understanding these mechanisms may form the basis of novel therapeutic approaches.
\end{abstract}

Key words: cytokines, adhesion molecules, acute myeloid leukemia, TNF-alpha, FLT3-ITD

Received: April 24, 2015; Accepted with revision: July 10, 2015; Available online: September 4, 2015

http://dx.doi.org/10.5507/bp.2015.036

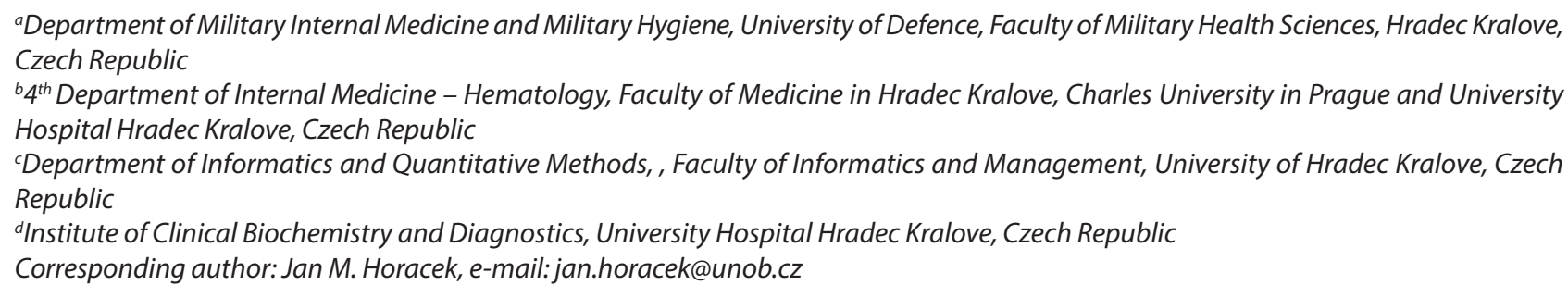

\section{INTRODUCTION}

The outcomes of AML treatment are beleaguered by the high resistance of malignant clones to therapy. Even high-dose multi-agent chemotherapy and allogeneic stem cell transplantation often fail to prevent relapse. Further, the toxicity of these regimens and the enormous risk of infection during prolonged pancytopenia prevent further dose escalation. Cytokines and adhesion molecules have been studied as markers of immune system activation in many diseases including AML. Experimentally, leukemic cytokines enhance endothelial cell growth in a co-culture of AML and endothelial cells ${ }^{1}$. There is evidence that AML activity influences serum levels of cytokines, growth factors and soluble adhesion molecules and promotes angiogenesis ${ }^{2,3}$. Tumor necrosis factor alpha (TNF- $\alpha$ ) and other cytokines are involved in the pathogenesis of acute myeloid leukemia (AML) and high-risk myelodysplastic syndromes (MDS). TNF- $\alpha$ secretion by AML cells and its ' involvement in the activation of signal transduction pathways and disease progression have been reported ${ }^{4,5}$. In a prospective study on newly diagnosed AML patients, lower TNF- $\alpha$ levels were associated with higher rates of complete remission and better event-free survival (EFS) in univariate analyses. However, in multivariate analyses, higher TNF- $\alpha$ levels were not found to be an independent factor predicting clinical outcome but became statistically significant, when leukocyte count was excluded from analyses ${ }^{6}$. AML cells were found to trigger positive feedback loops and activate endothelial cells. Activated endothelial cells express adhesion molecules involved in leukocyte rolling (P- and E-selectin) leukocyte adhesion (VCAM-1, ICAM-1), the same as chemoattractants MCP-1 and IL-8 (ref. ${ }^{7,8}$ ). Endothelial cell activation by TNF- $\alpha$ was followed by concomitant induction of VCAM1, ICAM-1 and E-selectin expression and time-dependent increase in the number of myeloblasts attached to the endothelium 9 . These interactions form the molecular basis of life threatening leukostasis and extramedular spread of AML. Further, adhesion to activated endothelia may dramatically increase the resistance of AML to chemotherapy and negatively influence treatment outcome ${ }^{10}$. Soluble (s) forms of adhesion molecules arise from proteolytic cleavage of surface-expressed molecules ${ }^{11}$. Increased binding of 
sVCAM-1 to $\alpha_{4} \beta_{1}$ integrin VLA-4 (Very Late Antigen-4) on myeloblasts was significantly associated with longer overall survival in newly diagnosed adult AML patients ${ }^{12}$. In conclusion, AML cell susceptibility to chemotherapy is modulated by dynamic adhesive interactions. To address this issue, we searched for links between pro-inflammatory cytokines, soluble adhesion molecules and other factors important for clinical decisions in newly diagnosed AML patients.

\section{PATIENTS AND METHODS}

\section{Patients}

A total of 53 newly diagnosed AML patients in the period 2010-2014, 32 with primary AML and 21 with secondary disease, were studied. Further details about patient cohort are summarized in Table 1. The group of secondary AML patients consisted of 17 patients with progression from myelodysplastic syndrome, 2 patients with progression from chronic myelomonocytic leukemia, 1 patient with previous primary myelofibrosis and 1 patient with unclassifiable myeloproliferation. Only patients eligible for induction chemotherapy were included. All patients were tested for cytogenetics and presence of molecular genetic aberrations. Twelve patients were Famosinlike tyrosin kinase 3 - internal tandem duplications (FLT3-ITD) positive. A total of 13 patients underwent leukapheresis due to initial hyperleukocytosis (Leucocyte count $\left.\geq 50 \times 10^{9} / \mathrm{L}\right)$. Patients with acute promyelocytic leukemia were treated with PETHEMA regimen combining Idarubicin and all-trans retinoic acid (ATRA). All other patients were induced with " $3+7$ ” induction chemotherapy consisting of Cytarabin $100 \mathrm{mg} / \mathrm{m}^{2}$ per day for 7 consecutive days and Daunorubicin $90 \mathrm{mg} / \mathrm{m}^{2}$ for the first 3 days of therapy in younger patients. In patients aged $\geq 65$ years, Daunorubicin $45-60 \mathrm{mg} / \mathrm{m}^{2}$ was administered. Seven patients had initial infection requiring intravenous antibiotic treatment. Complete remission (CR) was evaluated according to NCCN criteria. The study was approved by the local Ethics Committee and performed in accordance with the current Helsinki Declaration. All patients gave their written consent to the study.

\section{Serum collection}

Peripheral blood was collected in serum separating tubes, immediately transported to the laboratory and processed within $2 \mathrm{~h}$ of collection by centrifugation at $1500 \mathrm{rpm}$ x $5 \mathrm{~min}$. All sera were collected before leukapheresis, administration of hydroxyurea or induction therapy. If specimens were not to be analysed immediately, they were stored frozen in small aliquots at below $-70{ }^{\circ} \mathrm{C}$ as recommended by the Cytokine Array manufacturer. Repeat freeze/thaw cycles were avoided.

\section{METHODS}

All analytes were measured by biochip array technology using chemiluminescent sandwich immunoas-

Table 1. Characteristics of patients.

\begin{tabular}{|c|c|c|c|}
\hline & All patients & Primary AML & Secondary AML \\
\hline Patients & 53 & $32(60 \%)$ & $21(40 \%)$ \\
\hline Age (years) & $53.1 \pm 13.4$ & $46.3 \pm 13.3$ & $63.4 \pm 6.4$ \\
\hline \multicolumn{4}{|l|}{ Sex } \\
\hline Male & $20(38 \%)$ & $14(44 \%)$ & $6(29 \%)$ \\
\hline Female & $33(62 \%)$ & $18(56 \%)$ & $15(71 \%)$ \\
\hline \multicolumn{4}{|l|}{ Cytogenetics (risk) } \\
\hline Favourable & 11 & 11 & 0 \\
\hline Intermediate & 22 & 11 & 11 \\
\hline Adverse & 20 & 10 & 10 \\
\hline White blood cell count $\left(\times 10^{9} / \mathrm{L}\right)$ & $35.6 \pm 39.3$ & $46.8 \pm 50.9$ & $18.4 \pm 19.1$ \\
\hline Hyperleukocytosis & $13(25 \%)$ & $10(31 \%)$ & $3(14 \%)$ \\
\hline \multicolumn{4}{|l|}{ FAB type } \\
\hline M0 & 3 & 1 & 2 \\
\hline M1 & 11 & 6 & 5 \\
\hline M2 & 25 & 13 & 12 \\
\hline M3 & 4 & 4 & 0 \\
\hline M4 & 6 & 5 & 1 \\
\hline M5 & 3 & 3 & 0 \\
\hline M7 & 1 & 0 & 1 \\
\hline Active infection & $7(13 \%)$ & $3(9 \%)$ & $4(19 \%)$ \\
\hline CRP (mg/L) & $47.1 \pm 48.4$ & $39.3 \pm 37.1$ & $59.0 \pm 68.2$ \\
\hline FLT3-ITD & $12(21 \%)$ & $10(31 \%)$ & $2(9 \%)$ \\
\hline No CR after induction & $12(21 \%)$ & $4(13 \%)$ & $8(38 \%)$ \\
\hline
\end{tabular}


Table 2. Bonferroni-Holm corrected $P$ values of Spearman correlations for cytokines $\&$ adhesion molecule levels with age, leukocyte count and CRP. IL-8 and IL-10 not shown.

\begin{tabular}{|c|c|c|c|c|c|c|c|c|c|c|c|c|c|c|c|c|c|c|c|c|c|}
\hline & -2 & IL-4 & IL-6 & EGF & INF-G & NF-A & IL-1A & IL-1B & MCP-1 & EGF & VCAM-1 & ICAM-1 & E-SEL & P-SEL & IL-3 & IL-7 & IL-12 & |I-13 & Age & Leu & CRP \\
\hline IL-2 & & nen & 000 & 000 & 0000 & 000 & 709 & 601 & 000 & 0000 & 000 & 0000 & 0000 & 0000 & 000 & 000 & 0000 & 0000 & 0000 & .0000 & 0000 \\
\hline IL-4 & 000 & & 0000 & No & 00 & 000 & 509 & 00 & 00 & 0 & 00 & 00 & 00 & 000 & 00 & 00 & 000 & 000 & 00 & 00 & $n$ \\
\hline IL-6 & 000 & 0000 & & 0 & 00 & 00 & 0000 & 0000 & 00 & & 000 & 00 & 00 & 0 & 00 & 000 & 00 & 000 & 00 & 00 & 00 \\
\hline VEGF & 0000 & 0000 & 0000 & & 0000 & 0000 & 0000 & 0000 & 0000 & 120 & 000 & 0000 & 00 & 44 & 0000 & 3800 & 000 & 0000 & 0000 & 00 & .0000 \\
\hline INF-G & 0000 & 0000 & 0000 & 000 & & 0000 & 0000 & 1.0000 & 0.0386 & 0000 & 0000 & 1.0000 & 9462 & 1.0000 & 1.0000 & 1.0000 & 0000 & 1.0000 & .0000 & 1.0000 & 1.0000 \\
\hline TNF-A & 1.0000 & 0000 & 0000 & 0000 & 0000 & & 1.0000 & 1.0000 & 1.0000 & 1.0000 & 0.0035 & 0.0609 & 0.2495 & 1.0000 & 1.0000 & 1.0000 & 1.0000 & 1.0000 & 0000 & 0.5601 & 1.0000 \\
\hline IL-1A & 0.1709 & 6609 & 0000 & 0000 & 1.0000 & 0000 & & 06025 & 1.0000 & 1.0000 & 1.0000 & 1.0000 & 1.0000 & 1.0000 & 1.0000 & 1.0000 & 1.0000 & 1.0000 & 1.0000 & 1.0000 & 1.0000 \\
\hline IL-1B & 5601 & 0000 & 0000 & 0000 & 1.0000 & 1.0000 & 0.6025 & & 10000 & 1.0000 & 1.0000 & 1.0000 & 1.0000 & 1.0000 & 1.0000 & 1.0000 & 1.0000 & 1.0000 & 1.0000 & 1.0000 & 1.0000 \\
\hline MCP-1 & 9000 & 0000 & 0000 & 0000 & 0.0386 & 1.0000 & 1.0000 & 1.0000 & & 10000 & 1.0000 & 1.0000 & 0.0119 & 1.0000 & 1.0000 & 0.5102 & 1.0000 & 1.0000 & 1.0000 & 0.2029 & 1.0000 \\
\hline EGF & 000 & 0000 & 0000 & 0.0020 & 0000 & 1.0000 & 1.0000 & 1.0000 & 1.0000 & & $m$ & 1.0000 & 1.0000 & 00 & 1.0000 & 1.0000 & 1.0000 & 0000 & 00 & 1.0000 & 1.0000 \\
\hline VCAM-1 & 0000 & 0000 & 0000 & 0000 & 0000 & 0.0035 & 1.0000 & 1.0000 & 1.0000 & 1.0000 & & 0000 & 0.0000 & 0.2029 & 1.0000 & 1.0000 & .0000 & 0000 & 00 & 0.0005 & .0000 \\
\hline ICAM-1 & 0000 & 0000 & 0000 & 0000 & 1.0000 & 0.0609 & 1.0000 & 1.0000 & 1.0000 & 1.0000 & 000 & & 0.2026 & 00 & 1.0000 & 1.0000 & 00 & 0000 & 00 & 0.1011 & 3842 \\
\hline E-SEL & 1.0000 & 0000 & 0000 & 0000 & 462 & 495 & 1.0000 & 1.0000 & 0.0119 & 1.0000 & 0.0000 & 0.2026 & & 0.0032 & 1.0000 & 1.0000 & .0000 & .0000 & 0 & 10 & .0000 \\
\hline P-SEL & 1.0000 & 0000 & 0000 & 5044 & 0000 & 0000 & 1.0000 & 1.0000 & 1.0000 & 1.0000 & 0.2029 & 1.0000 & 0.0032 & & .0000 & 1.0000 & 1.0000 & .0000 & 00 & 1.0000 & 1.0000 \\
\hline IL-3 & 1.0000 & 0000 & 0000 & 0000 & 1.0000 & 1.0000 & 1.0000 & 1.0000 & 1.0000 & 1.0000 & 1.0000 & 1.0000 & 1.0000 & 1.0000 & & 1.0000 & 0.0604 & 1719 & .0000 & 1.0000 & 1.0000 \\
\hline IL-7 & 1.0000 & 0000 & 0000 & 3800 & 1.0000 & 1.0000 & 1.0000 & 1.0000 & 0.5102 & 1.00 & 1.0000 & 1.0000 & 1.0000 & 1.0000 & 1.0000 & & & .0000 & .0000 & & 1.0000 \\
\hline IL-12 & 1.0000 & 0000 & .0000 & .0000 & 1.0000 & 1.0000 & 1.0000 & 1.0000 & 1.0000 & 1.0000 & 1.0000 & 1.0000 & 1.0000 & 1.0000 & 0.0604 & 1.0000 & & 0993 & 0000 & 1.0000 & .0000 \\
\hline IL-13 & 1.0000 & .0000 & .0000 & .0000 & 1.0000 & 1.0000 & 1.0000 & 1.0000 & 1.0000 & 1.0000 & 1.0000 & 1.0000 & 1.0000 & 1.0000 & 0.1719 & 1.0000 & 0.0993 & & .0000 & 1.0000 & 1.0000 \\
\hline Age & 1.0000 & 1.0000 & 1.0000 & 1.0000 & 1.0000 & 1.0000 & 1.0000 & 1.0000 & 1.0000 & 1.0000 & 1.0000 & 1.0000 & 1.0000 & 1.0000 & 1.0000 & 1.0000 & 1.0000 & .0000 & & 1.0000 & 1.0000 \\
\hline Leu & 1.0000 & 1.0000 & 1.0000 & 1.0000 & 1.0000 & 0.5601 & 1.0000 & 1.0000 & 0.2029 & 1.0000 & 0.0005 & 0.1011 & 0.0000 & 1.0000 & 1.0000 & 0.0318 & 1.0000 & 1.0000 & 1.0000 & & 1.0000 \\
\hline CRP & 1.0000 & .0000 & 0.0000 & 1.0000 & 1.0000 & 1.0000 & 1.0000 & 1.0000 & 1.0000 & 1.0000 & 1.0000 & 0.3842 & 1.0000 & 1.0000 & 1.0000 & 1.0000 & 1.0000 & 1.0000 & 1.0000 & 1.0000 & \\
\hline
\end{tabular}

says applied to the Evidence Investigator Analyzer (Randox Laboratories Ltd., Crumlin, UK). The Evidence Investigator Biochip Array technology is used to perform simultaneous quantitative detection of multiple analytes from a single patient sample. The core technology is the Randox Biochip, a solid-state device containing an array of discrete test regions of immobilised antibodies specific to different cytokines and growth factors. A sandwich chemiluminescent immunoassay was employed for the cytokine array. Increased levels of cytokine in a specimen will lead to increased binding of antibody labelled with horseradish peroxidase (HRP) and thus an increase in the chemiluminescent signal emitted. The light signal generated from each of the test regions on the biochip is detected using digital imaging technology and compared to that from a stored calibration curve. The concentration of analyte present in the sample is calculated from the calibration curve.

We evaluated circulating levels of the following $17 \mathrm{cy}$ tokines and 5 soluble adhesion molecules: interleukins (IL-1 $\alpha$, IL-1 $\beta$, IL-2, IL-3, IL-4, IL-6, IL-7, IL-8, IL-10, IL12p70, IL-13, IL-23), vascular endothelial growth factor (VEGF), tumor necrosis factor-alpha (TNF- $\alpha)$, interferongamma (IFN- $\gamma)$, epidermal growth factor (EGF), monocyte chemotactic protein-1 (MCP-1), E-selectin, L-selectin, P-selectin, intercellular adhesion molecule-1 (ICAM-1) and vascular cell adhesion molecule-1 (VCAM-1). The results are expressed in nanograms per litre (ng/L) for cytokines and micrograms per litre $(\mu \mathrm{g} / \mathrm{L})$ for adhesion molecules.

\section{Statistical analysis}

Statistical analysis was performed in the R statistical programming environment, version 3.1.2 (http://www.Rproject.org). The levels of L-selectin were at the upper limit of the Array sensitivity in the majority of patients with hyperleukocytosis, similar to several patients with- out hyperleukocytosis. The levels of IL-23 were below the threshold limits for the majority of patients and hence were not included in further analyses. We analysed correlations between levels of cytokines and soluble adhesion molecules and associations with age, leukocyte count, secondary AML, CRP, FLT3-ITD and remission. We also searched for association of these variables and the first principal component (PC1). Spearman correlations were used pair-wise for continuous variables. To test the association between dichotomous and continuous variables the Mann-Whitney U test (ties-adjusted version from $\mathrm{R}$ package coin) was used ${ }^{13}$. Fisher's exact test was used for pairs of categorical variables. The Bonferroni-Holm correction ${ }^{14}$ was applied where necessary. In all analyses, $P<0.05$ was considered statistically significant.

\section{RESULTS}

\section{Cytokines and soluble adhesion molecules levels}

The levels of VCAM-1 strongly correlated with ICAM-1 $(r=0.67)$ and E-selectin $(r=0.64)$ levels (in both cases with corrected $P<0.0001$ ). The correlation with P-selectin was moderate and was not significant after Bonferroni-Holm correction. The E-selectin correlated with P-selectin $(r=0.56)$ and ICAM-1 $(r=0.44)$, but only correlation with P-selectin was significant with correction $P=0.0032$. There were significant correlations between several cytokines and adhesion molecule levels. TNF- $\alpha$ moderately correlated with VCAM-1 $(r=0.56$, corrected $P=0.0035)$ and ICAM-1 $(\mathrm{r}=0.48$, corrected $P=0.0609$ ), correlations with E- or P-selectin were not significant. MCP-1 was moderately negatively correlated with E-selectin $(\mathrm{r}=-0.53)$ and positively correlated with IFN- $\gamma(\mathrm{r}=0.50)$, corrected $P=0.0119$ and $P=0.0386$ respectively. The levels of EGF and VEGF correlated 
(corrected $P=0.002)$. The correlation of IL-12 with IL-3 and IL-13 was close to statistical significance $(P=0.0604$; $P=0.0993)$ after Bonferroni-Holm correction, the same as the correlation of IL- $1 \alpha$ and IL-4 $(P=0.0609)$. The CRP levels strongly correlated with IL-6 levels with $r=0.67$, corrected $P=0.0001$. There was no significant correlation of any evaluated analyte with age in this dataset. For all details see Table 2.

\section{Leukocyte count}

The leukocyte count correlated strongly with levels of VCAM-1 and E-selectin, which was significant after Bonferroni-Holm correction with $\mathrm{p}=0.0005$ for VCAM-1 and $P<0.0001$ for E-selectin respectively. The levels of IL-1 $\beta(P=0.0048)$ and E-selectin $(P=0.0158)$ were increased in hyperleukocytosis. The negative correlations of leukocyte count with IL-7 and MCP-1 were moderate, but for IL-7 this was still significant after Bonferroni-Holm correction $(P=0.0318)$. No other significant correlations between any two continuous variables were found.

\section{FLT3-ITD}

The FLT3-ITD mutation was present in 12 cases. The leukocyte count was associated with FLT3-ITD mutation, which remained close to statistical significance after Bonferroni-Holm correction with $P=0.0614$. In the presence of FLT3-ITD, the E-selectin (corrected $P=$ 0.0010, Fig. 1), and to lesser extent P-selectin (corrected $P=0.0770$, data not shown) were increased, whereas IL-7 was decreased (corrected $P=0.0252$ ). IL-1 $\beta$ was not associated with FLT3-ITD. FLT3-ITD was more frequent in primary AML cases but it was not statistically significant (data not shown). We found no significant association between FLT3-ITD mutation and CR attainment after induction therapy.

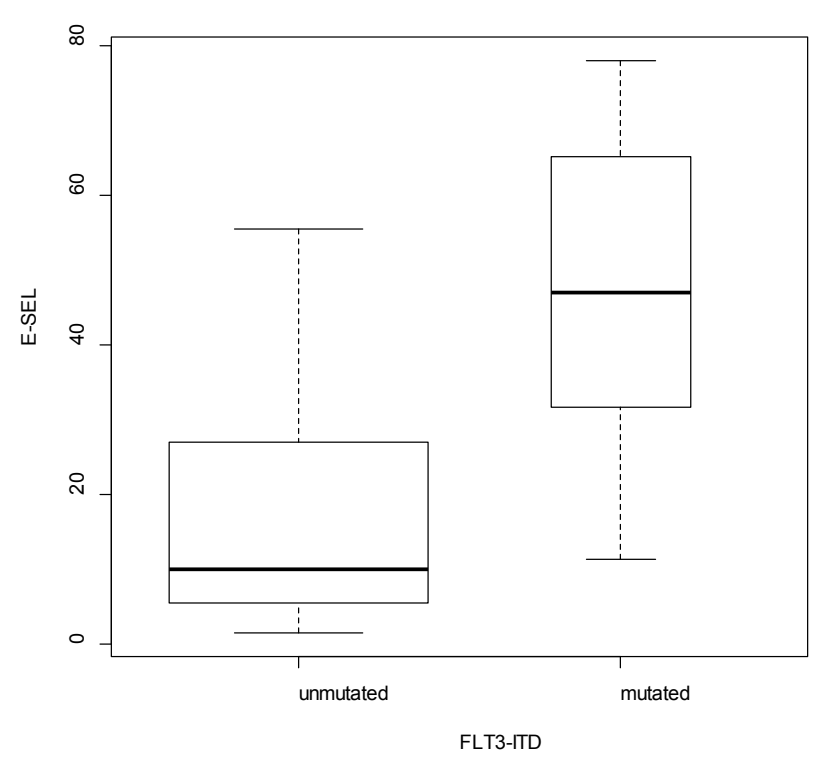

Fig. 1. The association of FLT3-ITD mutation and E-selectin, $P=0.0010$.

\section{Secondary AML and CR attainment}

Secondary origin of AML was significantly associated with higher age (corrected $P=0.0008$ ). The IL-10 was decreased in secondary AML but not significantly after Bonferroni-Holm correction. Other analytes evaluated in this dataset were not significantly associated with secondary AML origin. The secondary AML cases had less CR rates than primary cases but not significantly after Bonferroni-Holm correction. Failure of induction therapy was associated with higher CRP levels and lower P-selectin levels which were not significant after Bonferroni-Holm correction corrected $P=0.1032$ and $P$ $=0.1035$ respectively.
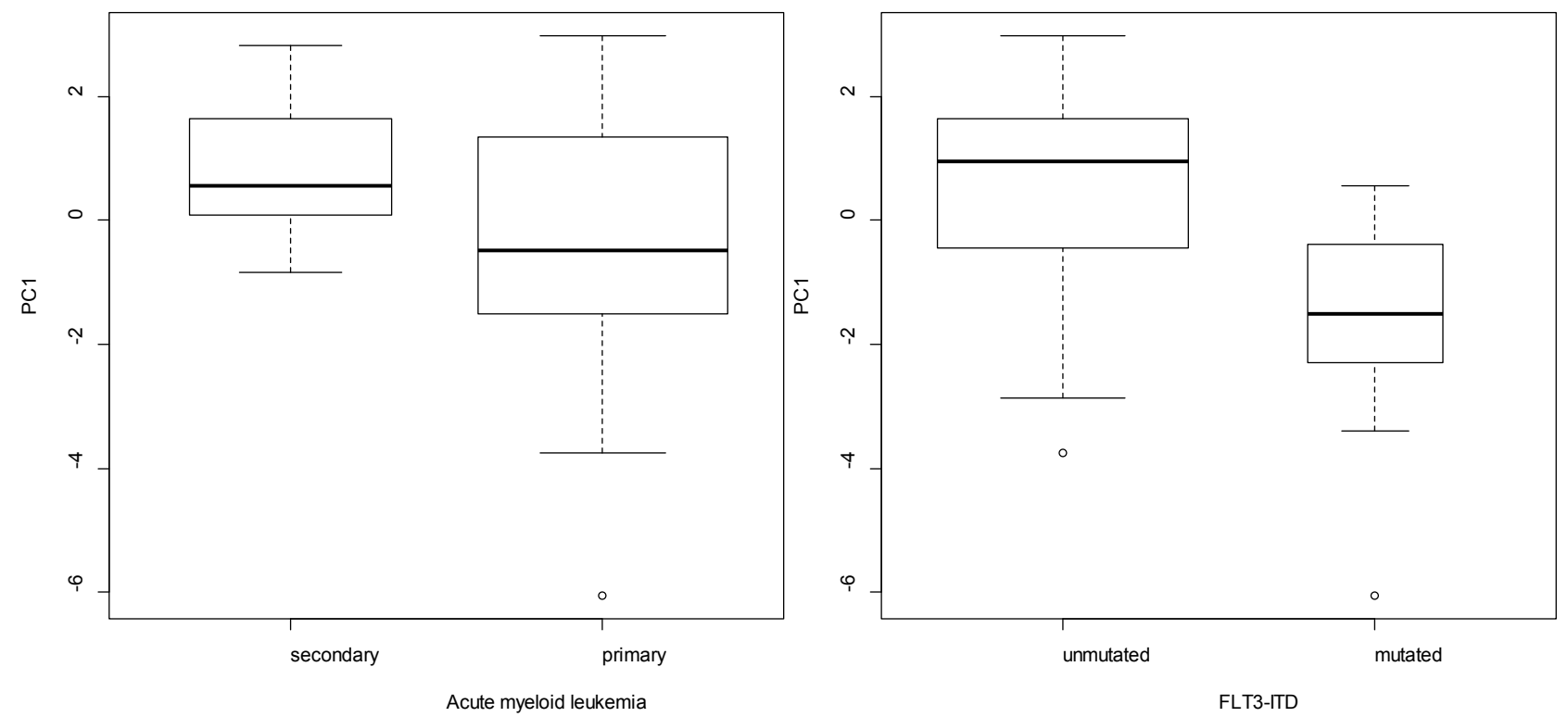

Fig. 2. The association of PC1 with FLT3-ITD $(P=0.0017)$ and secondary AML origin $(P=0.0439)$. 


\section{Principal component analysis}

The first principal component (PC1) explained 17\% of the variation in the cytokine and adhesion molecule levels. The PC1 significantly correlated with leukocyte count ( $\mathrm{r}=-0.71$, corrected $P=0.0001)$. FLT3-ITD mutation status (corrected $P=0.0017$, Fig. 2) and secondary AML origin (corrected $P=0.0439$, Fig. 2) also influenced PC1 significantly. Age, CR attainment and CRP levels had no significant influence on PC1.

\section{DISCUSSION}

Altered levels of cytokines and adhesion molecules have been linked to autoimmune diseases, allergies and cancer, including AML (ref. ${ }^{15,16}$ ). An inflammatory environment is asscociated with tumor progression ${ }^{15}$ and this shows that tumors are dynamic, interacting systems. Overexpression of cytokines in acute myeloid leukemia patients declines after chemotherapy administration or remission ${ }^{2,17}$. TNF- $\alpha$ has a pluripotent role in oncogenesis. Supportive care for some cancers, but not AML so far, includes inhibition of TNF signalling through the use of soluble receptors and neutralizing antibodies ${ }^{18,19}$. In vitro experiments published so far demonstrate that cytokines and adhesion molecules form a unique interacting functional network. As we lacked data on cytokine and adhesion molecule interplay in vivo, we decided to study both systems together in newly diagnosed AML patients. We used serum for this study as recommended by the Array manufacturer. It has been observed that levels of 44 of 80 cytokines, chemokines, and growth factors are significantly different in serum and plasma ${ }^{20}$.

Our findings are in agreement with the results of studies showing that cytokine and chemokine expression are largely independent of variables, such as age, gender and haemoglobin levels ${ }^{21}$. On the other hand, we found no correlation of the TNF- $\alpha$ levels and leukocyte counts that have been described previously ${ }^{6}$. We also failed to identify any significant changes in cytokine or adhesion molecule levels associated with standard risk stratification of AML. What we consider important is the finding that adhesion molecule levels are influenced as a whole and correlate with leukocyte count and TNF- $\alpha$ levels. TNF- $\alpha$ triggered and p38 MAPK driven endothelial cell activation was demonstrated in a model of leukostasis and inflammatory bowel disease ${ }^{7,9}$. We found significant positive correlation of TNF- $\alpha$ and VCAM-1, which in vivo, confirmed our expectation of its pivotal role in endothelial cell activation. To the best of our knowledge, this is the first study evaluating the interplay of an inflammatory environment and adhesion molecules in AML patients. On the other hand, leukocyte count significantly correlated with VCAM-1 and E-selectin but not with TNF- $\alpha$, so there are undoubtedly other factors involved in endothelial cell activation either singly or in combination, e.g. IL-1 $\beta$. Both IL-1 $\beta$ and E-selectin were increased in hyperleukocytosis. IL-1 $\beta$ was found to stimulate OCI/AML3 cell line proliferation in an autocrine manner ${ }^{22}$.
We consider the changes associated with FLT3-ITD partially reflect the increased leukocyte count. E-selectin was increased in FLT3-ITD positive cases and correlated with leukocyte count, which was almost significantly higher $(P=0.0614)$ in FLT3-ITD positive cases. So the FLT3-ITD seems to be another factor predicting endothelial cell activation. We suppose the FLT3-ITD cells are highly active and able to modulate the interplay between AML cells, cytokines and endothelial cell overexpression of adhesion molecules. We conclude both TNF- $\alpha$ and the activity of FLT3-ITD positive AML cells are key factors involved in endothelial cell activation. The results of the PC1 analysis support this conclusion. To the best of our knowledge, the interplay between cytokine, adhesion molecules and specific mutation in AML has not been studied so far. At this point, we have to admit that our results are based on analysis of a quite heterogeneous group of 53 AML patients. We consider this study a pivotal trial in this field. To provide more evidence further studies in a larger cohort of patients should be performed.

In this dataset, we failed to identify any cytokine or adhesion molecule associated significantly with CR attainment. On the other hand, we speculate that CRP and P-selectin in predicting CR could be informative in a larger cohort of patients. CRP levels strongly and significantly correlated with IL-6. Some leukemic cells were confirmed to constitutively express IL-6 with possible autocrine or paracrine receptor stimulation ${ }^{23}$. Activation of IL-6 signal transduction involves dimerization of IL-6 receptor gp130 subunit, consequently recruitment of gp130-associated protein-tyrosine kinases Jak1, Jak2, and Tyk2 and tyrosine phosphorylation of STAT-3, to a lesser extent STAT-1. Constitutive STAT-3 activation has been demonstrated in AML $\left(\right.$ ref. $^{24}$ ). Thus, in some AML cases CRP levels need not reflect active infection but they also serve as markers of natural disease activity. In one study, the presence of infection correlated with IL-8 levels (nominal $P=0.01)$ but this was not significant after Bonferroni correction $^{21}$. What seems to be the most troublesome is the fact that one can never be sure about infection status of newly diagnosed patients. The presence of infection, either apparent or obscure, is frequent in these patients. In our dataset, especially secondary AML patients had frequent infections at the time of diagnosis. This may limit the use of cytokine and adhesion molecule levels as prognostic markers.

What raises concern is the influence of secondary disease origin, but not of age on PC1. Secondary AML is more frequent in older patients. Nevertheless, due to progress in the treatment of malignancies in younger patients (e.g. Hodgkin 's lymphoma, testicular, early breast and prostate cancer) and curative treatment, younger patients also suffer from secondary AML. In a recently published study on adult AML patients, the IL-6 levels inversely and IL-10 levels directly correlated with survival ${ }^{25}$. CRP levels were not evaluated in that study. We found IL-10 nonsignificantly decreased in secondary AML. Our results indicate that these findings might be connected. 
Acknowledgement: The work was supported by a specific research project "Analysis of defined prognostic factors in acute myeloid leukemia" (Faculty of Military Health Sciences in Hradec Kralove), a long-term organisation development plan 1011 (Faculty of Military Health Sciences in Hradec Kralove) and a project for conceptual development of research organization 00179906 (Ministry of Health, Czech Republic).

Author contributions: TK: literature search; TK, JMH: research design; MV, IK: laboratory procedures; JV, TK: statistical analysis, TK, JMH: manuscript writing; PZ, LJ, JMH: critical revision.

Conflict of interest statement: The authors state that there are no conflicts of interest regarding the publication of this article.

\section{REFERENCES}

1. Hatfield K, Øyan AM, Ersvaer E, Kalland KH, Lassalle P, Gjertsen BT, Bruserud $\varnothing$. Primary human acute myeloid leukaemia cells increase the proliferation of microvascular endothelial cells through the release of soluble mediators. Br J Hematol 2009;144(1):53-68.

2. Horacek JM, Vasatova M, Kupsa T, Jebavy L, Zak P. Multi-analytica evaluation of serum levels of cytokines and adhesion molecules in patients treated for acute myeloid leukemia using biochip array technology. Biomed Pap Med Fac Univ Palacky Olomouc Czech Repub 2013;157(4):277-9.

3. Hatfield KJ, Evensen L, Reikvam H, Lorens JB, Bruserud $\varnothing$. Soluble mediators released by acute myeloid leukemia cells increase capillary-like networks. Eur J Haematol 2012;89(6):478-90.

4. Girgis EH, Mahoney JP, Rafaat KH, Soliman MR. Effect of thalidomide and arsenic trioxide on the release of tumor necrosis factor-a and vascular endothelial growth factor from the KG-1a human acute myelogenous leukemia cell line. Oncology letters 2010;1(4):663-7.

5. Volk A, Li J, Xin J, You D, Zhang J, Liu X, Xiao Y, Breslin P, Li Z, Wei W, Schmidt R, Li X, Zhang Z, Kuo PC, Nand S, Zhang J, Chen J, Zhang J. Co-inhibition of NF-KB and JNK is synergistic in TNF-expressing human AML. J Exp Med 2014;211(6):1093-108.

6. Tsimberidou AM, Estey E, Wen S, Pierce S, Kantarjian H, Albitar M, Kurzrock R. The prognostic significance of cytokine levels in newly diagnosed acute myeloid leukemia and high-risk myelodysplastic syndromes. Cancer 2008;113(7):1605-13.

7. Scaldaferri F, Sans M, Vetrano S, Correale C, Arena V, Pagano N, Rando G, Romeo F, Potenza AE, Repici A, Malesci A, Danese S. The role of MAPK in governing lymphocyte adhesion to and migration across the microvasculature in inflammatory bowel disease. Eur J Immuno 2009;39(1): 290-300.

8. Zarbock A, Ley K. Neutrophil adhesion and activation under flow. Microcirculation 2009;16(1): 31-42.

9. Stucki A, Rivier AS, Gikic M, Monai N, Schapira M, Spertini O. Endothelial cell activation by myeloblasts: molecular mechanisms of leukostasis and leukemic cell dissemination. Blood 2001;97(7):21219.

10. Pezeshkian B, Donnelly C, Tamburo K, Geddes T, Madlambayan GJ. Leukemia Mediated Endothelial Cell Activation Modulates Leukemia Cell Susceptibility to Chemotherapy through a positive Feedback
Loop Mechanism. Plos One 2013; 8(4):e60823. doi:10.1371/journal. pone.0060823.

11. Morandini R, Ghanem G, Portier-Lemarié A, Robaye B, Renaud A, Boeynaems JM. Action of CAMP on expression and release of adhesion molecules in human endothelial cells. Am J Physiol 1996;(7)270:807-16.

12. Becker PS, Kopecky KJ, Wilks AN, Chien S, Harlan JM, Willman CL, Petersdorf SH, Stirewalt DL, Papayannopoulou T, Appelbaum FR. Very late antigen- 4 function of myeloblasts correlates with improved overall survival for patients with acute myeloid leukemia. Blood 2009;113(4): 866-74.

13. Hothorn T, Hornik K, van de Wiel MA, Zeileis A. Implementing a Class of Permutation Tests: The coin Package. Journal of Statistical Software 2008;28(8):1-23.

14. Holm S. A simple sequentially rejective multiple test procedure. Scandinavian Journal of Statistics 1979;6(2):65-70.

15. Coussens LM, Werb Z. Inflammation and cancer. Nature 2002;420(6917):860-7.

16. Kupsa T, Horacek JM, Jebavy L. The role of cytokines in acute myeloid leukemia: a systematic review. Biomed Pap Med Fac Univ Palacky Olomouc Czech Repub 2012;156(4):291-301.

17. Hsu H, Lee Y, Tsai W, Jiang ML, Ho CH, Ho CK, Wang SY. Circulating levels of thrombopoietic and inflammatory cytokines in patients with acute myeloblastic leukemia and myelodysplastic syndrome. Oncology 2002;63(1):64-9.

18. Egberts JH, Cloosters V, Noack A, Schniewind B, Thon L, Klose S, Kettler B, von Forstner C, Kneitz C, Tepel J, Adam D, Wajant H, Kalthoff $\mathrm{H}$, Trauzold A. Anti-tumor necrosis factor therapy inhibits pancreatic tumor growth and metastasis. Cancer Res 2008;68(5):1443-50.

19. Popivanova BK, Kitamura K, Wu Y, Kondo T, Kagaya T, Kaneko S, Oshima M, Fujii C, Mukaida N. Blocking TNF-alpha in mice reduces colorectal carcinogenesis associated with chronic colitis. J Clin Invest 2008; $118(2): 560-70$.

20. Stroncek D, Slezak S, Khuu H, Basil C, Tisdale J, Leitman SF, Marincola FM, Panelli MC. Proteomic signature of myeloproliferation and neutrophilia: analysis of serum and plasma from healthy subjects given granulocyte colony-stimulating factor. Exp Hematol 2005:33(10):1109-17.

21. Kornblau SM, McCue D, Singh N, Chen W, Estrov Z, Coombes KR. Recurrent expression signatures of cytokines and chemokines are present and are independently prognostic in acute myelogenous leukemia and myelodysplasia. Blood 2010;116(20):4251-61.

22. Estrov Z, Black RA, Sleath PR, Harris D, Van Q, LaPushin R, Estey EH, Talpaz M. Effect of interleukin-1 beta converting enzyme inhibitor on acute myelogenous leukemia progenitor proliferation. Blood 1995;86(12):4594-602.

23. Dokter WH, Tuyt L, Sierdsema SJ, Esselink MT, Vellenga E. The spontaneous expression of interleukin- 1 beta and interleukin- 6 is associated with spontaneous expression of AP-1 and NF-kappa B transcription factor in acute myeloblastic leukemia cells. Leukemia 1995;9(3):425-32.

24. Gouilleux-Gruart V, Gouilleux F, Desaint C, Claisse JF, Capiod JC, Delobel J, Weber-Nordt R, Dusanter-Fourt I, Dreyfus F, Groner B, Prin L. STAT-related transcription factors are constitutively activated in peripheral blood cells from acute leukemia patients. Blood 1996;87(5):1692-7.

25. Sanchez-Correa B, Bergua JM, Campos C, Gayoso I, Arcos MJ, Bañas H, Morgado S, Casado JG, Solana R, Tarazona R. Cytokine profiles in acute myeloid leukemia patients at diagnosis: survival is inversely correlated with IL-6 and directly correlated with IL-10 levels. Cytokine 2013;61(3):885-91. 